\title{
An Efficient Adaptive based Median Technique to De-noise Colour and Greyscale Images
}

\author{
Gourav \\ Chandigarh Group of College/CSE, Mohali, Punjab,India \\ Email: Chalotragourav@gmail.com \\ Tejpal Sharma \\ Chandigarh Group of College /CSE, Mohali,Punjab,India \\ Email: tejpal.coecse@cgc.edu.in
}

Received: 24 November 2017; Accepted: 10 January 2018; Published: 08 February 2018

\begin{abstract}
The picture noise is an irregular variation of brightness and color information in pictures. It decreases picture quality and permeability of specific elements inside the picture. The most surely understood noise that corrupts the photo with impulse noise. In this work, an effective algorithm is intended to identify and remove noise from a picture. An improved de-noising calculation in view of the median filter is exhibited for greyscale and colored images. The algorithm incorporates two cases: I) if the chose window contains all pixel values " 0 " to "255" at that point center preparing pixel supplanted by the mean of qualities. II) If the chosen window does not contain all components " 0 " and "255" then eliminate "0" and " 255 " and central preparing pixel is replaced by the median of remaining pixels values. The performance is checked off the purposed algorithm by comparing it with corresponding filters. The experiment checked at various noise proportion $5 \%$ to $80 \%$ for greyscale and color pictures. Results are checked as far as MSE and PSNR and even at high noise proportion; it gives better outcomes over other existing techniques.
\end{abstract}

Index terms-Image features, Noise types and Filters, Algorithms steps, Performance analysis.

\section{INTRODUCTION}

Image de-noising is a standout amongst essential ideas in the figuring field. It is broadly utilized as a part of different picture related applications like MRI investigation, 3-D question discovery and so on. The digital pictures contain some level of noise. The objective of image de-noising is to re-establish the points of interest of a picture by evacuating undesirable noise. The de-noised picture ought not to contain noise. The noise brings arbitrary varieties into a picture that vacillate the first esteems to some unique esteems [1]. Causes which may acquaint noise with image incorporate blemishes in information transmission, flawed optics, sensor breaking down, handling systems and electronic impedance. Salt and pepper noise is a sort of impulse noise which is normally seen in pictures. It speaks to itself as arbitrarily happening white and dark pixels [2]. A photo containing this sort of noise will have dull pixels in splendid areas and wonderful pixels in diminish regions

\section{A. Application of de-noising}

- Picture de-nosing is used as a piece of the field of photography or circulating where a photo was somehow adulterated.

- It is utilized to make up for mutilation in the optical arrangement of a telescope.

- Image de-noising discovers applications in fields, for example, space science, therapeutic field.

- Used in legal science where conceivably valuable photographic confirmation is now and then off to a great degree of bad quality.

\section{B. Related work}

Ashwani et.al [3] presents a calculation for de noising in light of center channel is proposed. It has been actualized for the de-noising of dark scale and shading pictures which are debased by drive noise. This middle filter based calculation evaluates the uproarious pixels by middle esteem or with the assistance of aggregate investigation of the handled neighboring pixel esteems. The issue of expanding window size is additionally expelled. It additionally keeps the substitution of one boisterous information point with another loud information point rather than a middle esteem, and additionally, the proposed calculation, can sustain the reasonable middle if there are more than one maxima or the middle itself is contaminated.

Irum et.al [4] nonlinear half-breed channel for evacuating settled motivation commotion (salt and pepper) noise from shading pictures and made out of an arrangement of morphological standard and surely understood operations disintegration widening and trimmed standard middle channel. It expels the settled motivation commotion (salt and pepper) extremely well without contorting the picture highlights, shading segments, and edges. This Method uses the reverse property of the most rudimentary administrators of 
scientific morphology to be specific disintegration and enlargement.

Narayanan et.al [5] presents an effective noise evacuation method to re-establish computerized pictures defiled by blended noise, safeguarding picture content ideally and this separating strategy comprises of two stages: the uproarious pixel discovery step utilizing fluffy procedure and the blended noise sifting step. Clamour tended to in this methodology is a blend of salt and pepper bustle and Gaussian commotion. This technique lessens blended commotions impressively without trading off nervous sharpness. The proposed system reliably beats many existing fluffy based calculations while adjusting the tradeoffs between commotion diminishment and detail safeguarding.

Kulkarni et.al [6] presents exact and proficient noise recognition and separating calculation for motivation commotion evacuation. The calculation incorporates two phases: noise location taken after by commotion sifting. The proposed count replaces the uproarious pixel by cutout centre regard when another pixel regards, 0's or 255's are accessible in the picked window and when all the pixel regards are 0's and 255's then the clamor pixel is supplanted by the mean estimation of the significant number of segments display in the chose window and figuring is fruitful for salt and pepper hullabaloo clearing in pictures at high upheaval densities.

Malik et.al [7] presents a way to deal with the issue of commotion evacuation in color pictures and change in the two-sided channel which considers the closeness between shading pixels and their spatial separation. The cost of an association through an advanced way joining the focal pixel of the sifting window with the rest of the pixels is resolved. The yield, as in the standard two-sided channel, is calcium-recorded as a weighted normal of the pixels encompassing the focal point of the separating window and the weights are elements of the negligible association costs.

\section{De-noising methods}

\section{a) Types of image noise:}

Typical pictures are ruined with added substance noise displayed with either a Gaussian, speckle or salt or pepper noise. Another run of the spike noise is a spot noise, which is multiplicative in nature. Noise is available in a picture either in an added substance or multiplicative frame.

\section{b) Impulse Noise (Salt \& Pepper Noise):}

Salt and pepper commotion is a kind of fuss usually seen on pictures it astoundingly addresses itself as randomly happening white and dim pixels. A viable noise lessening approach for this sort of clamor includes the use of a middle channel or a differentiation symphonious mean filter. Salt and pepper noise impacts into pictures in circumstances where the picture is exchanged rapidly. The point of these techniques is to distinguish edges and subtle elements by methods for nearby measurements and smooth them not as much as whatever remains of the picture to better safeguard their sharpness[8]. Be that as it may, these techniques regularly recognize Impulses as points of interest or edges to be safeguarded.

\section{c) Speckle Noise:}

Speckle noise is a multiplicative clamor. This kind of commotion happens in all lucid imaging frameworks, for example, laser, acoustics, and SAR(Synthetic Aperture Radar) symbolism. The wellspring of this commotion is credited to the irregular impedance between the sound returns. Completely created spot clamor has the normal for the multiplicative commotion.

\section{d) Gaussian noise:}

Gaussian commotion is measurable noised having a likelihood thickness work (PDF) equivalent to that of the typical conveyance, which is otherwise, called the Gaussian distribution [9].

\section{De-noised filter methods}

\section{a) Adaptive filtering method:}

Adaptive Filtering Method [4] is a "choice-based" or "exchanging" channel with the reason for first recognizes conceivable loud pixels and after that supplant CES them utilizing the middle channel or its variations, however leaving every single other pixel unaffected. This channel is great at identifying commotion even at a far over the ground noise level. The flexible structure of this channel ensures with the desire of most of the inspiration bustles are recognized even at a far over the ground commotion level outfitted with the explanation behind the window estimate is sufficiently immense [9], [10]. The execution of AMF is great at subordinate noise thickness levels, because of the way that here are just less tainted pixels that are supplanted by the middle values.

\section{b) Median filtering:}

It is the least complex system which is utilized to expel dot clamor, beat commotion or Speckle noise from the picture. While other smoothing channels just expel noise from the flag yet they are not ready to protect edges of the flag but rather the middle channel is the extraordinary smoothing channel which gives better outcome by commotion expulsion from the flag alongside the safeguarding of the edges. In this method, pixel regard is supplanted by the focal point of the dim levels of the range of that pixel[11].

\section{c) Bilateral filtering method: -}

The bilateral filter is another non-direct separating technique which can be seen as an amplified adaptation of the low pass Gaussian sifting. Fundamentally, it is a basic course of action of a space channel, like the Gaussian channel, and a ranging channel which is a Gaussian capacity of nearby power contrast. The fundamental thought is that lone perceptually practically equivalent to hues are arrived at the midpoint of commonly to avoid the unpredicted shading blend in pictures. Barash bound together anisotropic dispersion and nonlinear two-sided sifting as an extra successful edge saving separating procedure [12]. 


\section{d) Vector median filters:}

These are principally intended for noise lessening in color images. There are different vector middle sifting methods, inside which the one called half and half vector separating is firmly identified with this work. A crossover channel uses various subs-channels of various sorts and characterizes the yield as a direct nonlinear blend of the information vectors. For instance, the developed vector middle channel consolidates the VMF with direct filtering [13]. Close edges, this channel carries on like the VMF, while in smooth territories it acts like the number juggling mean channel (AMF).

\section{PROPOSED FILTER FOR DE-NOISING}

The proposed an efficient adaptive based median technique has utilized the Restoration of color pictures. The pictures utilized are very debased by impulse commotion. The adaptive based median technique uses the already prepared neighboring pixel incentive to show signs of improvement picture quality. The algorithm has identified the pictures with impulse noise. In the first stage, the pixels in the picture are checked whether it is noisy or not. On the off chance that the preparing pixel lies between the greatest and least greyscale level range which is 0 and 255 , then it is without noise and left unaltered. On the off chance that the handling pixel takes the most extreme or least dark level then it is an uproarious pixel which is prepared by proposed algorithm.

\section{A. Algorithm steps:-}

1. The first step is to browse an image from the destination folder.

2. Add salt and pepper noise to the image according to noise distortion value.

3. Calculate the size of the noisy image.

4. Padded the noisy image matrix with zeros.

5. Select a window of size $3 * 3$. Assume that the central pixel is processed $P_{i j}$.

6. If center pixel $0<P_{i j}<255$, then $P_{i j}$ is left unchanged. As noise element are in range [0,255].

7. If $P_{i j}=0$ or $P_{i j}=255$ then choose for next condition. If $P_{i j}$ is corrupted pixel then two cases are possible as given in case I) if the selected window contains all the elements as 0 's and 255 's. Then " $P_{i j}$ " with the mean of the element of the window. As due to this the value of $P_{i j}$ comes in the range of 0-255.

Case II) If the selected window contains not all element as 0 's and 255 's. Then eliminate 255 's and 0 's and find the median value of remaining elements. Replace $P_{i j}$ with median value.

8. Repeat stages 5-8 until the point that every one of the pixels in the whole picture is handled.

9. Removal of extra padded rows and columns.

10. Calculate the PSNR and MSE. Show the enhanced picture.

\section{B. Clarification of the algorithm:-}

When a picture is taken as input then every pixel of a picture is checked for the presence of noise. In the event that the Pixels discovered noisily, then assume central pixels as " $P_{i j}$ "'is' and as pixels value is in range 0 to 255 . On the off chance that the central preparing pixel $P_{i j}$ lies between extents 0 to 255 then it will leave unaltered. In the event that the Processing Pixel is defined a pixel, then two cases outlined beneath:

Case1. If select Window consist of $3 * 3$ contains the whole component " 0 " and '255'. Select one incentive as handling pixels either "0" or "255" which is again noisy. To solve this problem, mean of pixels values are taken and replace it with the noisy pixel.

$$
\left[\begin{array}{ccc}
255 & 0 & 255 \\
0 & <255> & 255 \\
0 & 0 & 255
\end{array}\right]
$$

We found here mean of pixels esteems is 142 and supplant it with center processing pixel.

Case2. If in the event that the selected element does not contain all components " 0 " and "255" and have some other pixel value. At that point eliminate with " 0 " and "255" and locate the middle of other pixel values.

$$
\left[\begin{array}{ccc}
78 & 0 & 87 \\
65 & <0> & 66 \\
255 & 70 & 255
\end{array}\right]
$$

After the removal of noisy pixels " 0 " and "255," we have pixel value in the selected window $[65,66,70,78$, 87 ] and we have middle value is 70 which replaced with the center processing pixel ' $\mathrm{p}_{\mathrm{ij}}$ '.

\section{RESULTS AND DISCUSSION}

\section{A. Experimental images}

The Performance of the algorithm is assessed on a few genuine pictures appeared in Figure 1.These photos are the most generally utilized standard test pictures utilized for picture retargeting calculations and measurement of these pictures $512 \times 512$ in jpg format. With a particular ultimate objective to test the capability and the execution of the purposed filter and contrasting counts the examinations are performed at a bigger measure of a noise thickness, which goes from $05 \%$ to $80 \%$. These operations were performed on two-dimensional (2 - D) Greyscale pictures and color pictures.

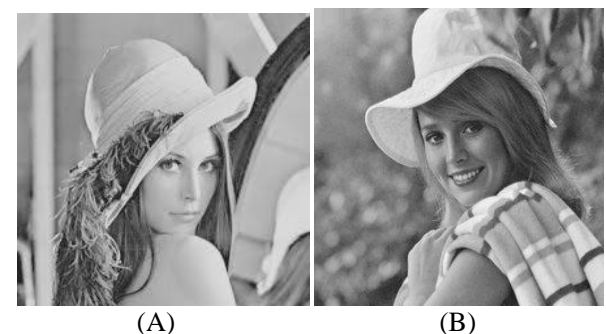

(A) (B) 


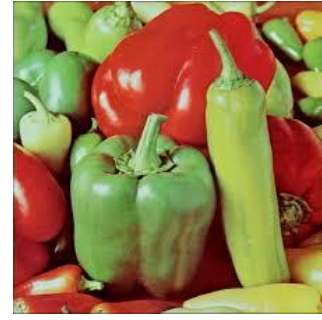

(C)

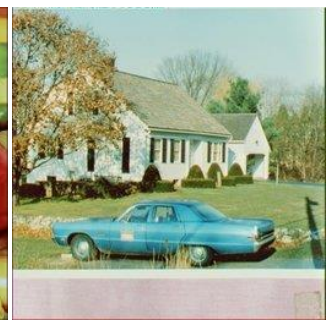

(D)
Fig.1. Experimental Images: (a) Image of Lena (b) Image of Elaine (c) Image of Pepperr (d) Image of house

Table 1. Experimental images result after applying purpose filter

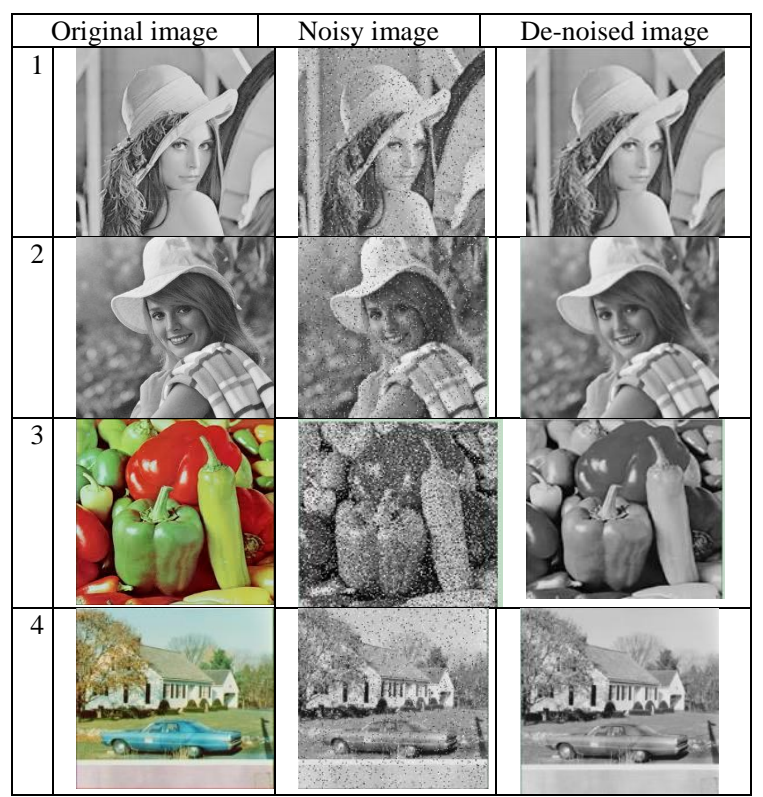

\section{A. Peak Signal-To-Noise-Ratio:}

PSNR is the proportion is the extent between the most extreme conceivable energy of a Signal and the energy of debasing noise that impacts the steadiness of its representation. PSNR high means great quality and low means awful quality. In this way, low the blunder, high will be the PSNR. In Mathematically PSNR is computed by:

$$
P S N R=10 \cdot \log _{10}+\frac{\mathrm{MAX}_{1}^{2}}{\mathrm{MSE}}
$$

Here, MAXI is the maximum possible pixel value of the image. When the pixels are represented using 8 bits per sample, this is 255 .

\section{B. Mean squared error (MSE):}

The mean square error is the normal of the squared blunders amongst real and assessed readings in an information test.

$$
\operatorname{MSE}=\frac{1}{M N}+\sum_{\mathrm{i}=0}^{\mathrm{m}-1} \quad \sum_{\mathrm{j}=0}^{\mathrm{n}-1}[\mathrm{I}(\mathrm{i}, \mathrm{j})-\mathrm{K}(\mathrm{i}, \mathrm{j})]^{2}
$$

\section{Performance Analysis}

The performance analysis of Impulse noise table has been evaluated, the Impulse noise ratio from 5\% to $80 \%$ iterations are evaluated for Barbara Image, steam community image, Tumblr images, greyscale image and color image.

\section{PERFORMANCE METRICS}

To evaluate the performance of the de-noising procedures a few execution measurements are accessible. We utilize the PSNR, Mean Square Error for various experimental images.

\begin{tabular}{|c|c|c|c|c|c|c|c|c|c|c|}
\hline \multirow[t]{2}{*}{ Filter } & \multirow[t]{2}{*}{ metrics } & \multicolumn{9}{|c|}{ Noise ratio } \\
\hline & & 0.05 & 0.1 & 0.2 & 0.3 & 0.4 & 0.5 & 0.6 & 0.7 & 0.8 \\
\hline \multirow{2}{*}{$\begin{array}{l}\text { Improved median } \\
\text { filter }\end{array}$} & MSE & 1.29 & 2.96 & 8.71 & 18.41 & 31.82 & 54.36 & 97.73 & 171.29 & 296.50 \\
\hline & PSNR & 47.24 & 43.28 & 38.48 & 35.40 & 32.99 & 30.99 & 28.31 & 26.03 & 23.59 \\
\hline \multirow{2}{*}{$\begin{array}{l}\text { Efficient adaptive } \\
\text { based median } \\
\text { technique }\end{array}$} & MSE & 0.88 & 1.28 & 3.42 & 6.77 & 11.97 & 26.99 & 75.48 & 114.22 & 259.41 \\
\hline & PSNR & 51.01 & 47.05 & 42.77 & 39.82 & 37.34 & 33.81 & 29.35 & 27.55 & 23.99 \\
\hline
\end{tabular}

Table 2. Performance analysis for salt and pepper noise for Lena image image

\begin{tabular}{|c|c|c|c|c|c|c|c|c|c|c|}
\hline \multirow[t]{2}{*}{ Filter } & \multirow[t]{2}{*}{ metrics } & \multicolumn{9}{|c|}{ Noise ratio } \\
\hline & & 0.05 & 0.1 & 0.2 & 0.3 & 0.4 & 0.5 & 0.6 & 0.7 & 0.8 \\
\hline \multirow{2}{*}{$\begin{array}{l}\text { Improved median } \\
\text { filter }\end{array}$} & MSE & 2.34 & 3.44 & 9.36 & 19.22 & 32.44 & 49.44 & 85.28 & 162.23 & 292.39 \\
\hline & PSNR & 35.33 & 34.11 & 34.61 & 33.73 & 32.30 & 30.82 & 29.49 & 28.49 & 28.07 \\
\hline \multirow{2}{*}{$\begin{array}{c}\text { Efficient adaptive } \\
\text { based median } \\
\text { technique }\end{array}$} & MSE & 0.20 & 0.54 & 1.60 & 3.18 & 6.78 & 16.37 & 49.34 & 115.24 & 200.41 \\
\hline & PSNR & 55.10 & 50.79 & 46.08 & 43.09 & 39.81 & 35.98 & 31.19 & 29.26 & 25.11 \\
\hline
\end{tabular}

Table 3. Performance analysis for salt and pepper noise for Elaine image 
Table 4. Performance analysis for salt and pepper noise for Pepper image

\begin{tabular}{|c|c|c|c|c|c|c|c|c|c|c|}
\hline \multirow[t]{2}{*}{ Filter } & \multirow[t]{2}{*}{ metrics } & \multicolumn{9}{|c|}{ Noise ratio } \\
\hline & & 0.05 & 0.1 & 0.2 & 0.3 & 0.4 & 0.5 & 0.6 & 0.7 & 0.8 \\
\hline \multirow{2}{*}{$\begin{array}{c}\text { Improved } \\
\text { method filter }\end{array}$} & MSE & 1.37 & 3.50 & 9.76 & 18.28 & 34.35 & 56.71 & 93.46 & 170.25 & 343.74 \\
\hline & PSNR & 46.73 & 42.94 & 38.50 & 35.50 & 33.18 & 30.66 & 28.34 & 25.45 & 23.49 \\
\hline \multirow{2}{*}{$\begin{array}{c}\text { Efficient } \\
\text { adaptive based } \\
\text { median } \\
\text { technique }\end{array}$} & MSE & 0.34 & 0.86 & 2.56 & 5.24 & 10.72 & 23.24 & 65.76 & 100.16 & 200.04 \\
\hline & PSNR & 52.50 & 48.77 & 43.55 & 40.99 & 37.82 & 34.46 & 29.95 & 28.12 & 24.15 \\
\hline
\end{tabular}

Table 5. Performance analysis for salt and pepper noise for House color image.

\begin{tabular}{|c|c|c|c|c|c|c|c|c|c|c|}
\hline \multirow[t]{2}{*}{ Filter } & \multirow[t]{2}{*}{ metrics } & \multicolumn{9}{|c|}{ Noise ratio } \\
\hline & & 0.05 & 0.1 & 0.2 & 0.3 & 0.4 & 0.5 & 0.6 & 0.7 & 0.8 \\
\hline \multirow{2}{*}{$\begin{array}{c}\text { Improved } \\
\text { median filter }\end{array}$} & MSE & 3.22 & 6.44 & 12.48 & 21.98 & 33.13 & 57.23 & 112.32 & 234.35 & 398.13 \\
\hline & PSNR & 42.23 & 40.76 & 37.49 & 34.22 & 31.44 & 29.55 & 27.26 & 26.22 & 23.48 \\
\hline \multirow{2}{*}{$\begin{array}{c}\text { Efficient } \\
\text { adaptive } \\
\text { based median } \\
\text { technique }\end{array}$} & MSE & 1.30 & 2.19 & 6.54 & 11.99 & 21.97 & 41.39 & 97.38 & 186.01 & 354.31 \\
\hline & PSNR & 47.98 & 44.72 & 39.97 & 37.34 & 34.71 & 31.96 & 28.24 & 27.21 & 24.01 \\
\hline
\end{tabular}

\section{CONCLUSION}

In this work, another calculation is presented which give better performance at higher noise ratio by comparing with their corresponding results. The performance of algorithm checked at various level of noise ratio for greyscale and color images which prove that it gives better performance than existing methods. During replacement of noisy pixels in the window, it does not affect the other pixels. Due to its capability to distinguishes the disturb pixels and afterward separates them from good ones. Although it gives better results at $80 \%$ of noise ratio and the purpose algorithm is effective for salty noise in the image at the low and high-level intensity.

\section{ACKNOWLEDGMENT}

Most importantly we thank we thank god-like God whose grace was there throughout the course of the paper. We might want to thank our parents and friends for their endless support. Finally like to express our deep sense of gratitude to our colleagues, Dept. Of computer \& Science, CGC Landran, Punjab for their enduring support and encouragement.

\section{REFERENCES}

[1] A. Kumar and R. Roi, "Algorithm for De-noising of Color Images based on Median Filter 1,2,4,5," pp. 428432, 2015.

[2] I. Irum, M. Sharif, M. Raza, and S. Mohsin, “A Nonlinear Hybrid Filter for Salt \& Pepper Noise Removal from Color Images," J. Appl. Res. Technol., vol. 13, no. 1, pp. 79-85, 2015.

[3] M. Jayasree and N. K. Narayanan, "An efficient mixed noise removal technique from grayscale images using noisy pixel modification technique," 2015 Int. Conf. Commun. Signal Process. ICCSP 2015, pp. 336-339, 2015.

[4] M. R. N. Kulkarni and P. P. C. Bhaskar, "Implementation of Decision-Based Algorithm for Median Filter to extract Impulse Noise," vol. 2, no. 6, pp. 2507-2512, 2013

[5] V. Murugan and T. Avudaiappan, "A Comparative
Analysis of Impulse Noise Removal Techniques on Gray Scale Images," vol. 7, no. 5, pp. 239-248, 2014.

[6] "IMPROVED BILATERAL FILTERING SCHEME FOR NOISE REMOVAL IN COLOR IMAGES Krystyna Malik , Bogdan Smolka Polish-Japanese Institute of Information Technology Bilateral Filter," pp. 2-8

[7] B. C. Patel and G. R. Sinha, "Gray-level clustering and contrast enhancement (GLC-CE) of mammographic breast cancer images," CSI Trans. ICT, vol. 2, no. 4, pp. 279-286, 2015.

[8] C.Anjanappa and H.S.Sheshadri, "Survey on Impulse Noise Removal in Digital Images," vol. 6, no. 7, pp. 4551, 2012.

[9] S. Ansari and K. Mangla, "Eliminating Noise from Mixed Noisy Image by using Modified Bilateral Filter," Ijarcet, vol. 4, no. 5, pp. 2327-2332, 2015.

[10] R. Soman and J. Thomas, "A Novel Approach for Mixed Noise Removal using ' ROR ' Statistics Combined WITH ACWMF and DPVM,” vol. 86, no. 17, pp. 11-17, 2014.

[11] V. Crnojević and N. Petrović, "Impulse Noise Filtering Using Robust Pixel-Wise S-Estimate of Variance," EURASIP J. Adv. Signal Process., vol. 2010, no. 1, p. 830702, 2010.

[12] M. J. Tanakian, M. Rezaei, and F. Mohanna, "Digital video stabilizer by adaptive fuzzy filtering," EURASIP J. Image Video Process., vol. 2012, no. 1, p. 21, 2012.

[13] S. Marukatat, "Image enhancement using local intensity distribution equalization," EURASIP J. Image Video Process., vol. 2015, no. 1, p. 31, 2015.

[14] T. M. Khan, M. A. U. Khan, Y. Kong, and O. Kittaneh, "Stopping criterion for linear anisotropic image diffusion: a fingerprint image enhancement case," EURASIP J. Image Video Process., vol. 2016, no. 1, p. 6, 2016.

\section{Authors' Profiles}

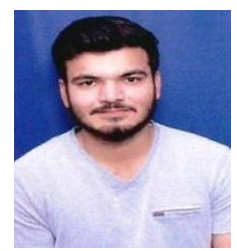

Gourav is currently pursuing M.tech in computer science from CGC-COE, Mohali, Punjab. He received his B.tech degree in computer science from S.S.C.E.T Badhani, Punjab in 2014 and presently working in the area of "Digital image processing" with his Asst. professor Tejpal Sharma. 


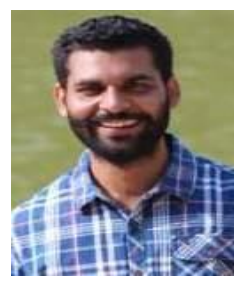

Tejpal Sharma presently pursuing Ph.D. on "digital image processing" from Punjabi university, Patiala and receive his M.tech degree from B.B.S.B Engineering College, Fatehgarh Sahib, Punjab.Currently working as an Assistant professor in Chandigarh group of College, Landran, Mohali and Punjab.

How to cite this paper: Gourav, Tejpal Sharma, " An Efficient Adaptive based Median Technique to De-noise Colour and Greyscale Images", International Journal of Modern Education and Computer Science(IJMECS), Vol.10, No.2, pp. 48-53, 2018.DOI: 10.5815/ijmecs.2018.02.06 